\title{
Dislocations: 75 Years of Deformation Mechanisms
}

\author{
J.A. Schneider
}

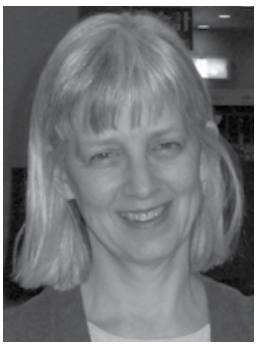

The selection of papers presented in this section reflect on themes to be explored at the Dislocations: 75 Years of Deformation Mechanisms Symposium to be held at the TMS 2009 Annual Meeting in February. The symposium is sponsored by the Mechanical Behavior of Materials Committee to give tribute to the evolution of a concept that has formed the basis of our mechanistic understanding of how crystalline solids plastically deform and how they fail. Historically and now, the advancement of dislocation theory continues to be based on complimentary analytical, conceptual, and experimental observations.

The utility of metals is based on a combination of stiffness, strength, and ductility, or the ability to be plastically deformed. It is the ductility that greatly expands the usefulness of metals by their ability to be produced in various shapes by processes such as pressing, rolling, forming, or forging. This is an area in which art, science, and technology are intertwined. Early metalworking and blacksmithing grew into the science of metallurgy without the benefit of a formalized theory of the physics or mechanisms responsible for deformation. Although science stayed at the periphery, discussions evolved sometimes simultaneously, to offer an explanation for the art and technology used to fabricate tools, weapons, bridges, and machines.

Individual manuscripts published by Taylor, Orowan, and Polanyi in 1934 independently offered theories to explain the deformation of ductile metals at stresses lower than that predicted by the bond strength. These concepts would take several decades to develop into dislocation theory which formally describes mechanisms of dislocation movement and impediment. Understanding of these mechanisms has laid the groundwork for design of highstrength alloys and retention of material strength at elevated temperatures.

\section{Understanding of}

\section{these mechanisms has}

laid the groundwork

for design of high-

strength alloys and

retention of material

strength at elevated

\section{temperatures.}

Dislocations are implicated in the failure of ductile metal, and failure is usually preceded by the growth and coalescence of voids. How the voids originate and grow is modeled using molecular dynamics in "The Role of Dislocations in the Growth of Nanosized Voids in Ductile Failure of Metals" by M. Meyers et al.

In "The Intermediate Temperature Deformation of Ni-based Superalloys: Importance of Reordering" by L. Kovarik et al., experimental efforts are combined with first-principle calculations of the reordering that may occur within the strengthening gamma prime precipitates.

Advances in our understanding of plasticity mechanisms advanced in par- allel with our understanding of failure mechanisms It is known that the temperature effects on plastic behavior in body-centered cubic metals are different from face-centered cubic metals. "Dislocation Motion in Phonon Liquids" by John J. Gilman summarizes how the earlier theories have been disproved as purer alloys have become available for testing. By evoking a fluid dynamics analogy, an updated explanation is offered for the differences in the flow stresses observed experimentally.

Recent advances in the understanding of rate-controlling mechanisms in precipitation-strengthened materials at various temperatures are considered in "Dislocation-Particle Interaction at Elevated Temperatures" by R. Mishra, who reviews the various mechanisms proposed for theoretical prediction of threshold stress and how they are affected by temperature.

Current research efforts continue to bridge the length scale between experimental observations and computational predictions and continuum modeling. "Dislocation Nucleation and Multiplication in Small Volumes: The Onset of Plasticity during Indentation Testing" by D.F. Bahr et al. discusses the advances in testing small volumes of material to explore incipient plasticity mechanisms.

These manuscripts are representative of the papers to be presented at the TMS 2009 Annual Meeting to be held February 15-19, in San Francisco, California. Additional information can be found at www.tms.org.

\footnotetext{
J.A. Schneider is with the Mechanical Engineering Department at Mississippi State University and is the JOM advisor from the Mechanical Behavior Committee of theTMS Structural Materials Division. She can be reached at schneider@me.msstate. edu.
} 